\title{
The high pressure structure and equation of state of 2,6-diamino-3,5-dinitropyrazine-1- oxide (LLM-105) up to 20 GPa: X-ray diffraction measurements and first principles molecular dynamics simulations
}

Cite as: J. Chem. Phys. 143, 144506 (2015); https://doi.org/10.1063/1.4932683

Submitted: 19 May 2015 . Accepted: 23 September 2015 . Published Online: 14 October 2015

Elissaios Stavrou, M. Riad Manaa, Joseph M. Zaug (D), I-Feng W. Kuo, Philip F. Pagoria, Bora Kalkan, Jonathan C. Crowhurst, and Michael R. Armstrong

ARTICLES YOU MAY BE INTERESTED IN

First-principles high-pressure unreacted equation of state and heat of formation of crystal 2,6-diamino-3, 5-dinitropyrazine-1-oxide (LLM-105)

The Journal of Chemical Physics 141, 064702 (2014); https://doi.org/10.1063/1.4891933

Equations of state of 2,6-diamino-3,5-dinitropyrazine-1-oxide

Journal of Applied Physics 110, 073523 (2011); https://doi.org/10.1063/1.3646492

The equation of state of 5-nitro-2,4-dihydro-1,2,4,-triazol-3-one determined via in-situ optical microscopy and interferometry measurements

Journal of Applied Physics 119, 135904 (2016); https://doi.org/10.1063/1.4945426
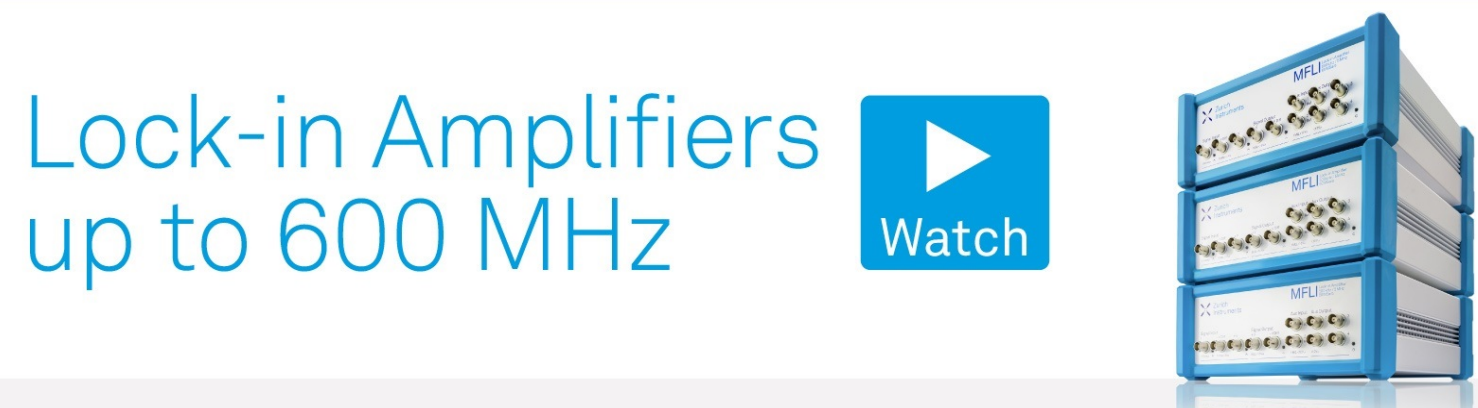

J. Chem. Phys. 143, 144506 (2015); https://doi.org/10.1063/1.4932683 


\title{
The high pressure structure and equation of state of 2,6-diamino-3,5-dinitropyrazine-1-oxide (LLM-105) up to $20 \mathrm{GPa}$ : $\mathrm{X}$-ray diffraction measurements and first principles molecular dynamics simulations
}

\author{
Elissaios Stavrou, ${ }^{1, a)}$ M. Riad Manaa, ${ }^{1, b)}$ Joseph M. Zaug, ${ }^{1}$ I-Feng W. Kuo, ${ }^{1}$ \\ Philip F. Pagoria, ${ }^{1}$ Bora Kalkan, ${ }^{2,3}$ Jonathan C. Crowhurst, ${ }^{1}$ and Michael R. Armstrong ${ }^{1}$ \\ ${ }^{1}$ Lawrence Livermore National Laboratory, Physical and Life Sciences Directorate, P.O. Box 808 L-350, \\ Livermore, California 94550, USA \\ ${ }^{2}$ Advanced Light Source, Lawrence Berkeley Laboratory, Berkeley, California 94720, USA \\ ${ }^{3}$ Advanced Materials Research Laboratory, Department of Physics Engineering, \\ Hacettepe University 06800, Beytepe, Ankara, Turkey
}

(Received 19 May 2015; accepted 23 September 2015; published online 14 October 2015)

\begin{abstract}
Recent theoretical studies of 2,6-diamino-3,5-dinitropyrazine-1-oxide $\left(\mathrm{C}_{4} \mathrm{H}_{4} \mathrm{~N}_{6} \mathrm{O}_{5}\right.$ Lawrence Livermore Molecule No. 105, LLM-105) report unreacted high pressure equations of state that include several structural phase transitions, between 8 and $50 \mathrm{GPa}$, while one published experimental study reports equation of state (EOS) data up to a pressure of $6 \mathrm{GPa}$ with no observed transition. Here we report the results of a synchrotron-based X-ray diffraction study and also ambient temperature isobaric-isothermal atomistic molecular dynamics simulations of LLM-105 up to $20 \mathrm{GPa}$. We find that the ambient pressure phase remains stable up to $20 \mathrm{GPa}$; there is no indication of a pressure induced phase transition. We do find a prominent decrease in $b$-axis compressibility starting at approximately $13 \mathrm{GPa}$ and attribute the stiffening to a critical length where inter-sheet distance becomes similar to the intermolecular distance within individual sheets. The ambient temperature isothermal equation of state was determined through refinements of measured X-ray diffraction patterns. The pressure-volume data were fit using various EOS models to yield bulk moduli with corresponding pressure derivatives. We find very good agreement between the experimental and theoretically derived EOS. (C) 2015 AIP Publishing LLC. [http://dx.doi.org/10.1063/1.4932683]
\end{abstract}

\section{INTRODUCTION}

Low sensitivity, thermal and shock, and high energy density are among the most important characteristics of a useful energetic high explosive material. 2,6-diamino3,5-dinitropyrazine-1-oxide $\left(\mathrm{C}_{4} \mathrm{H}_{4} \mathrm{~N}_{6} \mathrm{O}_{5}\right.$ Lawrence Livermore Molecule No. 105, LLM-105) stands out as a very promising candidate due to its low insult sensitivity and high energy output. Indeed, experimental studies reveal that LLM-105 (usually in the form of a plastic bonded-polymeric matrix formulation, e.g., with Kel F- $800^{1}$ ) sensitivity is between that of 1,3,5-triamino-2,4,6-trinitrobenzene (TATB) a highly insensitive explosive, and octahydro-1,3,5,7-tetranitro1,3,5,7-tetrazine (HMX), which is more sensitive. ${ }^{2}$ Additionally, the energetic content of LLM-105 is also between that of TATB and HMX (about 20\% above TATB and $15 \%$ below HMX). ${ }^{3}$ LLM-105 was first synthesized in $1998^{4}$ and it has attracted considerable experimental ${ }^{5-10}$ and theoretical ${ }^{11-14}$ interest for the aforementioned reasons. From these studies, a detonation velocity $>7500 \mathrm{~m} / \mathrm{s}$ (depending on the formulation ${ }^{9,15}$ ) and a detonation pressure $>30 \mathrm{GPa}$ have been reported. Moreover, LLM-105 remains stable up to $\approx 530 \mathrm{~K}$, where decomposition occurs at ambient pressure. $^{8}$

\footnotetext{
a)E-mail: stavrou1@1lnl.gov

b)E-mail: manaa1@1lnl.gov
}

Engineering design initiatives have also been focused to produce energetic micro- and nano-structured devices; and recently, one such project yielded LLM-105 microtubes. ${ }^{6}$

LLM-105 crystallizes in a monoclinic $P 2_{1} / n(14)$ space group structure (Fig. 1) with four formula units per unit cell $^{5}$ and its relatively high stability should arise from the existence of intra- ${ }^{12}$ and inter- ${ }^{16}$ molecular hydrogen $\mathrm{H} \cdots \mathrm{O}$ bonds. The high pressure structural behavior of LLM-105 has been studied both theoretically ${ }^{13,14}$ up to $\approx 50 \mathrm{GPa}$ (at $0 \mathrm{~K}$ ) and experimentally ${ }^{8}$ up to $5.5 \mathrm{GPa}$ (at room temperature). A series of structural phase transitions at $8,17,25$, and $42 \mathrm{GPa}$ have been theoretically proposed by Wu et al. ${ }^{14}$ based on irregular changes of lattice parameters. More recently, Manaa et al. ${ }^{13}$ concluded that the ambient pressure phase of LLM-105 remains stable up to $45 \mathrm{GPa}$. Both theoretical studies suggest that LLM-105 exhibits highly anisotropic compressibility, i.e., $a$ and $c$ axes are much stiffer than the $b$ axis. Because experimental results of LLM-105 were reported only up to a maximum $5.5 \mathrm{GPa}$, the issue of structural behavior at higher pressures must be re-examined and established. Moreover, an accurate determination of the room-temperature high-pressure unreacted equation of state (EOS) is essential for continuum based simulations of physical and mechanical properties including performance under loading conditions. ${ }^{13}$

In this work, we have conducted a detailed X-ray diffraction (XRD) study and a first principles room temperature 


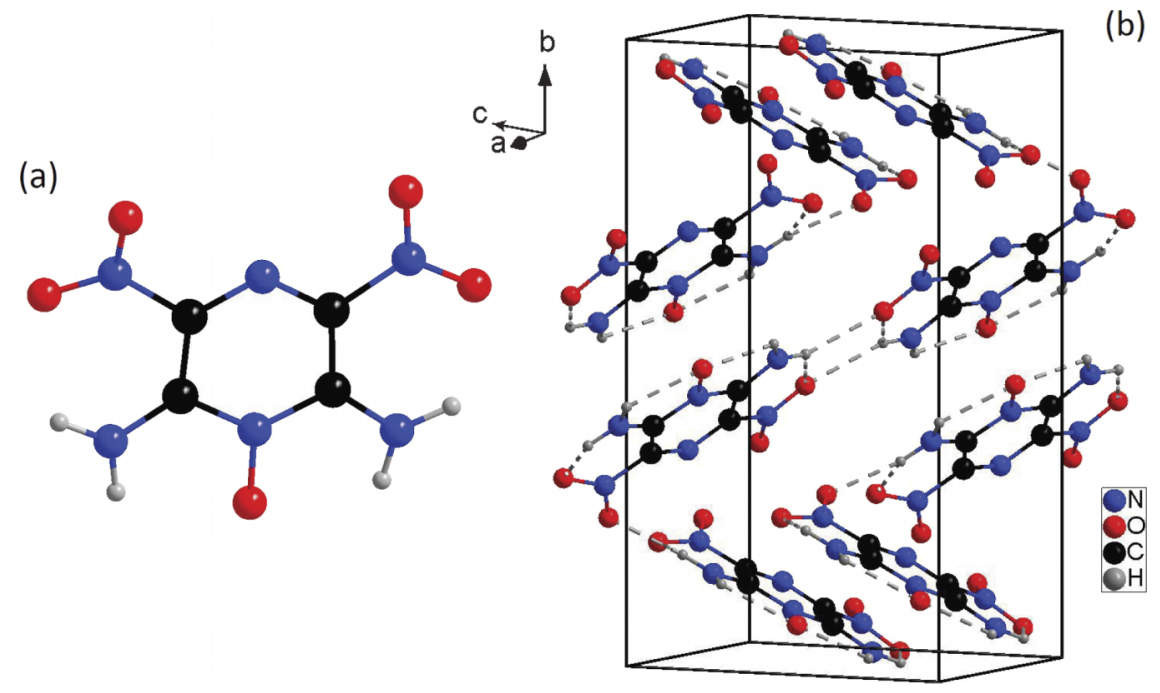

(b)

computational simulations of LLM-105 up to $20 \mathrm{GPa}$. We implemented dispersion-corrected density-functional theory (DFT) based isothermal-isobaric molecular dynamics simulations $(\mathrm{T}=300 \mathrm{~K})$ at various pressures for single crystal LLM105. We find from both the experimental and computational results that LLM-105 remains in the ambient pressure phase up to the highest pressure of this study without any sign of a structural phase transition. The simulation results are found to be in very good agreement with the experimentally determined EOS up to $20 \mathrm{GPa}$. It is established that LLM105 clearly exhibits anisotropic compression; up to $13 \mathrm{GPa}$, compressibility along the $b$-axis is measurably higher than along the $a$ and $c$ axes.

\section{METHODS}

\section{A. Experimental}

LLM-105 was synthesized with a $57 \%$ yield by the nitration of 2,6-diaminopyrazine-1-oxide (DAPO) with a mixture of $100 \% \mathrm{HNO}_{3}$ and $10 \%$ fuming sulfuric acid at $20-30{ }^{\circ} \mathrm{C}$ for $3 \mathrm{~h}$. Recrystallization was achieved by dissolving LLM-105 as a $2.5 \%$ solution in $\mathrm{N}, \mathrm{N}$-dimethylformamide (DMF) at $120^{\circ} \mathrm{C}$ and by slowly adding two volumes of warm water and allowing the mixture to cool to $\sim 40^{\circ} \mathrm{C}$. The LLM-105 crystal habit typically exhibits as brilliant yellow rods. Additional details of the synthesis procedure can be found in Ref. 4 .

LLM-105 single crystals were ground to a fine powder for x-ray diffraction measurements. The sample and pressure sensors (gold or ruby) were loaded into diamond-anvil cell (DAC) sample chambers. For each of two X-ray studies, rhenium gaskets (preindented to $40-45 \mu \mathrm{m}$ thick using $400 \mu \mathrm{m}$ culets) were used to radially confine the pressurized samples. Initial sample chamber diameters were nominally $50 \mu \mathrm{m}$. Silicone oil was utilized as a pressure-transmitting medium (PTM): this material is relatively inert, easy to load, and does not exhibit Bragg diffraction peaks. Silcone oil solidifies at nominally $13 \mathrm{GPa}^{17}$ at room temperature (RT); however, with a deviatoric stress of less than $2 \mathrm{GPa}$ at $20 \mathrm{GPa}$ pressure, ${ }^{18}$ we continue to consider it as a quasi-hydrostatic PTM. A MAR345 image plate detector was used to collect pressure dependent XRD data at the Advanced Light Source Beamline 12.2.2 (X-ray spot size is $\sim 10 \mu \mathrm{m} \times 10 \mu \mathrm{m}$ and $\lambda=0.4959 \AA$ ). To minimize the possibility of $\mathrm{X}$-ray induced decomposition, diffraction measurements were never repeated at the same spatial position within the sample. Pressure was determined using a known ambient temperature EOS of gold ${ }^{19}$ and also calibrated ruby luminescence. ${ }^{20}$ The maximum pressure uncertainty was less than $2 \mathrm{GPa}$ at the highest pressure achieved in this study. Powder diffraction patterns were integrated using the FIT2 $\mathrm{D}^{21}$ program to yield scattering intensity versus $2 \theta$ diagrams.

\section{B. Computational methods}

The reported calculations were performed using the description of our previous study, which determined the cold compression curve ( $\mathrm{T}=0 \mathrm{~K})$ of LLM-105, among other properties. ${ }^{13}$ Briefly, we use DFT within the generalized gradient corrected approximation of Perdew-Burke-Ernzerhof (PBE) for the exchange-correlation potential. ${ }^{22}$ Lack of dispersion interaction in DFT was corrected for with the inclusion of two-body (D2) dispersion correction as proposed by Grimme. ${ }^{23} \mathrm{~A}$ dual basis set formalism for the description of wavefunctions and for electron density was utilized. A triple zeta with double polarization (TZV2P) Gaussian type orbital basis was implemented for the wavefunctions, while plane wave basis expanded to $320 \mathrm{Ry}$ is used to represent the electron density. The core electronic states are represented by the norm conserving Goedecker-Teter-Hutter pseudopotentials. ${ }^{24}$ For cell geometry optimization, the following convergence criteria were implemented: total energy was converged to a tolerance of less than $1 \times 10^{-5} \mathrm{eV} /$ atom, the residual forces to less than $0.03 \mathrm{eV} / \AA$, the residual stress to less than $0.05 \mathrm{GPa}$, maximum displacement to less than $0.001 \AA$, and the selfconsistent field convergence criterion of $5 \times 10^{-7} \mathrm{eV} /$ atom. The isobaric-isothermal molecular dynamics simulation is carried out following the recipe outlined by Schmidt et al. ${ }^{25}$ The wavefunctions are explicitly minimized to $10^{-7}$ Hartree using the orbital transformation method for every dynamics step. ${ }^{26}$ Forces are then utilized to propagate the molecular dynamics simulation in the isothermal-isobaric ensemble (NPT) with 
a fixed time step of 0.5 fs. Simulations were conducted at $\mathrm{T}=300 \mathrm{~K}$, with temperature being controlled via individual Nosé-Hoover chains coupled to each degree of freedom with a coupling constant of $2000 \mathrm{~cm}^{-1} .^{27,28}$ The barostat characteristic frequency was set to $500 \mathrm{fs}$. The calculations were performed on a supercell of dimension $3 \times 1 \times 2$ (containing 24 LLM-105 molecules, 456 atoms) using the Quickstep module within the $\mathrm{CP} 2 \mathrm{~K}$ simulation suite ${ }^{29,30}$ No constraints were applied to the box shape effectively allowing all six lattice parameters to move independently of each other using one single barostat. On the other hand, each atom has 3 thermostats controlling its kinetic energy.

\section{RESULTS}

In Figure 2(a), the experimental ambient pressure XRD pattern of LLM-105 is presented together with the Le Bail refinement of the calculated pattern according to Ref. 5. An excellent agreement between these two patterns can be observed thus indicating the absence of solid impurities in the starting LLM-105 specimen. Slight differences of relative intensity are normally attributed to preferred orientation effects in the powder pattern. In Figure 3, selected pressure dependent X-ray powder diffraction patterns of LLM-105 are presented. All except one of the observed peaks in this pressure range can be indexed with the ambient phase monoclinic structure. The intense additional Bragg peak, indicated with red arrows in Figure 3, appears as a complete diffraction ring in 2D XRD images at very low pressures, e.g., $0.1 \mathrm{GPa}$. It shifts to larger angles (smaller d-spacing) with increased pressure and gradually decreases in relative intensity up to approximately $19 \mathrm{GPa}$ where it virtually disappears. While the very "clean" ambient pressure LLM-105 diffraction pattern (Fig. 2(a)) rules out the presence of a solid impurity, pressureinduced crystallization of a liquid impurity would result in more than one Bragg peak. The same peak has been also observed in a previous independent XRD study of LLM-105

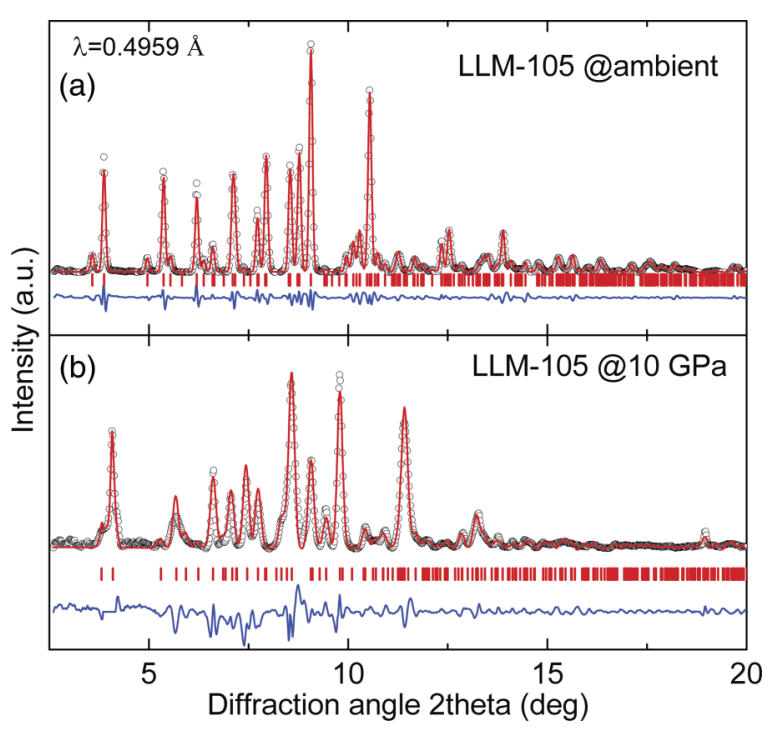

FIG. 2. Le Bail refinements of LLM-105 at (a) ambient pressure and (b) $10 \mathrm{GPa}$.

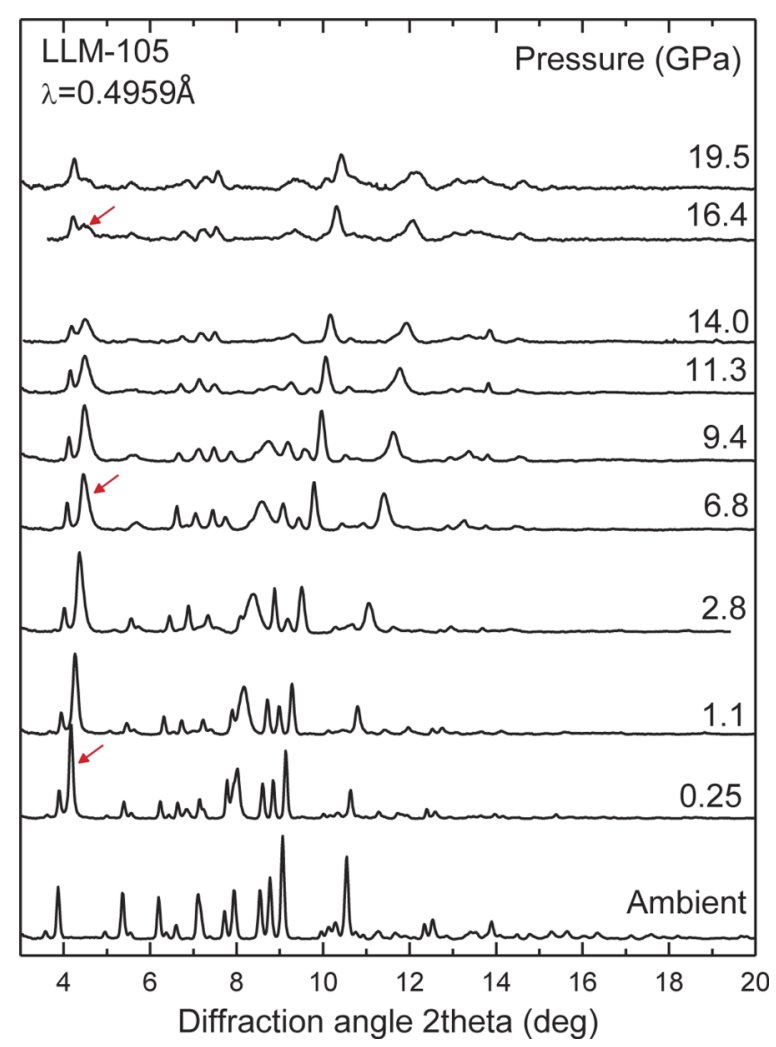

FIG. 3. Selected pressure dependent X-ray diffraction patterns of LLM-105. The red arrows indicate the Bragg peak that cannot be indexed with the ambient phase monoclinic structure.

under pressure. ${ }^{31}$ However, with the exception of this particular unassigned diffraction peak all other peaks up to $20 \mathrm{GPa}$ can be indexed using the ambient phase monoclinic structure. Thus, no first-order structural phase transition was detected up to $20 \mathrm{GPa}$. The diffraction patterns were analyzed by performing Le Bail refinements using the GSAS $^{32}$ software. A typical refined high-pressure profile is shown in Fig. 2(b). Example results of the Le Bail refinements together with the results of the theoretical calculations are summarized in Table I where EOS fit parameter values are also listed.

From NPT molecular dynamics simulation runs of up to approximately $11 \mathrm{ps}$, we have determined the cell parameters and volume at various pressure conditions and $\mathrm{T}=300 \mathrm{~K}$. We allowed an initial equilibration period of $1 \mathrm{ps}$ and discarded this period from our final analysis of the simulations results. In Figure 4, we present example time dependent profiles for results obtained at $\mathrm{P}=10 \mathrm{GPa}$. In the figure, the pressure is assumed/computed to be the trace of the stress tensor with the off-diagonal components proving to be very negligible. Figure 4 also has the temperature $(T=300 \mathrm{~K})$, the cell volume, and the lattice parameters temporal profiles. A final set of values at each pressure-temperature run of the cell parameters is obtained by using a block averaging procedure over the complete time period of the simulation, which also yields a standard deviation for the final reported value. A block size of $1 \mathrm{ps}$ was used in the statistical analysis.

The pressure dependent lattice parameters and unit cell volumes for the compression cycle are shown in Figures 5(a) and 5(b), respectively, together with the results of our room temperature theoretical calculations. A very good agreement 
TABLE I. Experimental data, from this study and from Ref. 5, and from this study calculated, lattice parameters and cell volumes of LLM-105 at selected pressures. Also listed are the bulk modulus, $\mathrm{K}_{0}$ and its pressure derivative $\mathrm{K}^{\prime}$ at zero pressure as determined by a third-order Birch-Murnaghan (B-M) equation of state unweighted fit.

\begin{tabular}{llllllll}
\hline \hline $\mathrm{P}(\mathrm{GPa})$ & & $\mathrm{a}(\AA)$ & \multicolumn{1}{c}{$\mathrm{b}(\AA)$} & $\mathrm{c}(\AA)$ & $\mathrm{V}$ cell $\left(\AA^{3}\right)$ & $\mathrm{K}(\mathrm{GPa})$ & $\mathrm{K}^{\prime}$ \\
\hline 0 & Expt. & $5.723(2)$ & $15.870(2)$ & $8.424(2)$ & $750.08(15)$ & $15(4)$ & $9(3)$ \\
0 & Expt. (Ref. 5) & 5.7159 & 15.8498 & 8.4139 & 748.16 & & \\
0 & Calc. & $5.81(8)$ & $16.05(9)$ & $8.40(5)$ & $769.51(14.36)$ & $12.7(4)$ & $9.4(3)$ \\
19.5 & Expt. & $5.15(8)$ & $13.62(12)$ & $7.74(8)$ & $542(5)$ & & \\
20 & Calc. & $5.29(3)$ & $13.3(3)$ & $7.72(2)$ & $535.79(3.92)$ & & \\
\hline \hline
\end{tabular}

between the two sets of data can be observed from the plots of Fig. 5: (a) $b$-axis as determined in this study is more compressible than $a$ and $c$ axes during initial compression and (b) the theoretically predicted EOS is in agreement with the experimental data. Consistent with most high-pressure EOS studies, we conducted unweighted fits of the pressure-volume data using a third-order Birch-Murnaghan (B-M) equation of state. ${ }^{33}$ The corresponding ambient condition bulk modulus $K_{0}$ and the first pressure derivative $K^{\prime}$ results are summarized in Table I.

To gain deeper insight into how LLM-105 responds under quasi-static compression, we conducted weighted fits and used the reduced $\chi_{\text {red }}^{2}$ goodness-of-fit formalism to compare the effectiveness of three EOS models to represent the $\mathrm{P}-\mathrm{V}$ data. The reduced $\chi_{\text {red }}^{2}$ value closest to 1 represents the "winning model." We tested the $\mathrm{B}-\mathrm{M}^{33} 2$ nd to 5 th orders, the Vinet, ${ }^{34}$ and the $\mathrm{F}-\mathrm{f}^{35}$ finite strain 1 st to $3 r d$ order EOS models. For each winning (best fit) model, where appropriate, we plot corresponding two-dimensional confidence ellipses to reveal two-variable correlation information. The winning model was determined to be the first-order F-f EOS; see Fig. 6. Bivariable confidence plots enable a more comprehensive basis for comparison of EOS parameters to alternative theoretical and/or experimental results. ${ }^{36}$ In this way, one can accurately assess if theoretical results are consistent with experimental results given a range of confidence. Application of the B-M model reveal that the third-order B-M yielded the statistically best representation of the data in compassion to the other $\mathrm{B}-\mathrm{M}$ orders. The $\chi_{\text {red }}^{2}$ value of the Vinet model is marginally better than the $\mathrm{B}-\mathrm{M}$ model; and, the Max $\Delta \mathrm{P}$ values are comparable. The F-f model gives the lowest $\chi_{\text {red }}^{2}$ values, the lowest maximum pressure deviation from the data and the
Kolmogorov-Smirnov test ${ }^{37,38}$ (KS-test) values closest to 0 . Refer to the Appendix for the complete statistical analysis of the fitting procedure. The fit results for the most optimal EOS models are summarized in Table II. The relatively high $\chi_{\text {red }}^{2}$ values from fits to the experimental data indicate that the assessed P-V errors are a bit low given the functional forms of the EOS models. Alternatively, other EOS models may exist that more optimally represent the data where $\chi_{\text {red }}^{2}$ values are closer to unity. Fits of the theoretical results, with very low $\chi_{\text {red }}^{2}$ values more clearly indicate that the assessed errors are a bit large (e.g., Vo) given the EOS functional forms.

\section{DISCUSSION}

As previously mentioned in the Introduction, a series of pressure induced phase transitions have been proposed by a previous theoretical study ${ }^{14}$ based on irregular changes of lattice parameters. These results suggest that isostructural phase transitions occur with possibly the same or closely related symmetry. Although no indication of a phase transition can be observed from XRD patterns up to the highest pressure of this study, which rules out a first-order phase transition, we further examine the possibility of a second order isostructural transition by performing a stress (normalized pressure $\left.=\mathrm{F}=\mathrm{P}\left[3 \mathrm{f}(1+2 \mathrm{f})^{5 / 2}\right]^{-1}\right)$-Eulerian strain $(\mathrm{f}=0.5$ $\left.\left[\left(\mathrm{V}_{0} / \mathrm{V}\right)^{2 / 3}-1\right]\right)(\mathrm{F}-\mathrm{f}) \mathrm{EOS}$ analysis ${ }^{40}$ (Fig. 6). The F-f EOS analysis is ideal to probe subtle (low volume discontinuities) structural changes, ${ }^{41}$ which are difficult to determine using P-V EOSs. ${ }^{41,42}$ Here the Vinet EOS parameters were used as initial guess value inputs for the F-f models. We observe that the pressure dependent stress increases linearly up to the

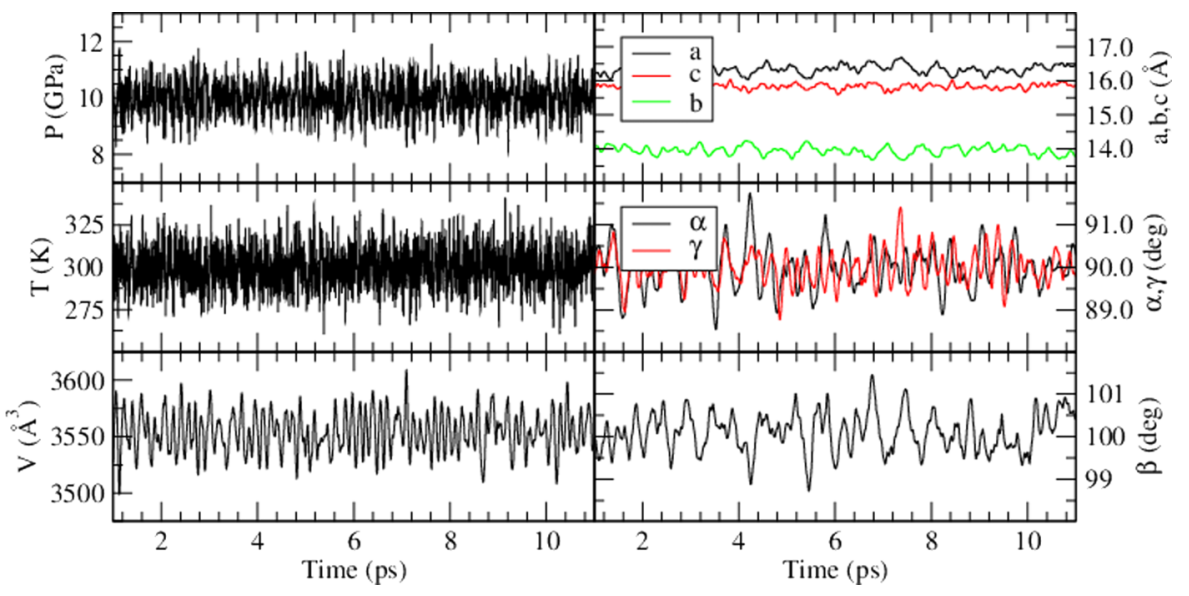

FIG. 4. A sample of computational results of total pressure, temperature, supercell volume, and supercell parameters at $\mathrm{P}=10 \mathrm{GPa}$. 

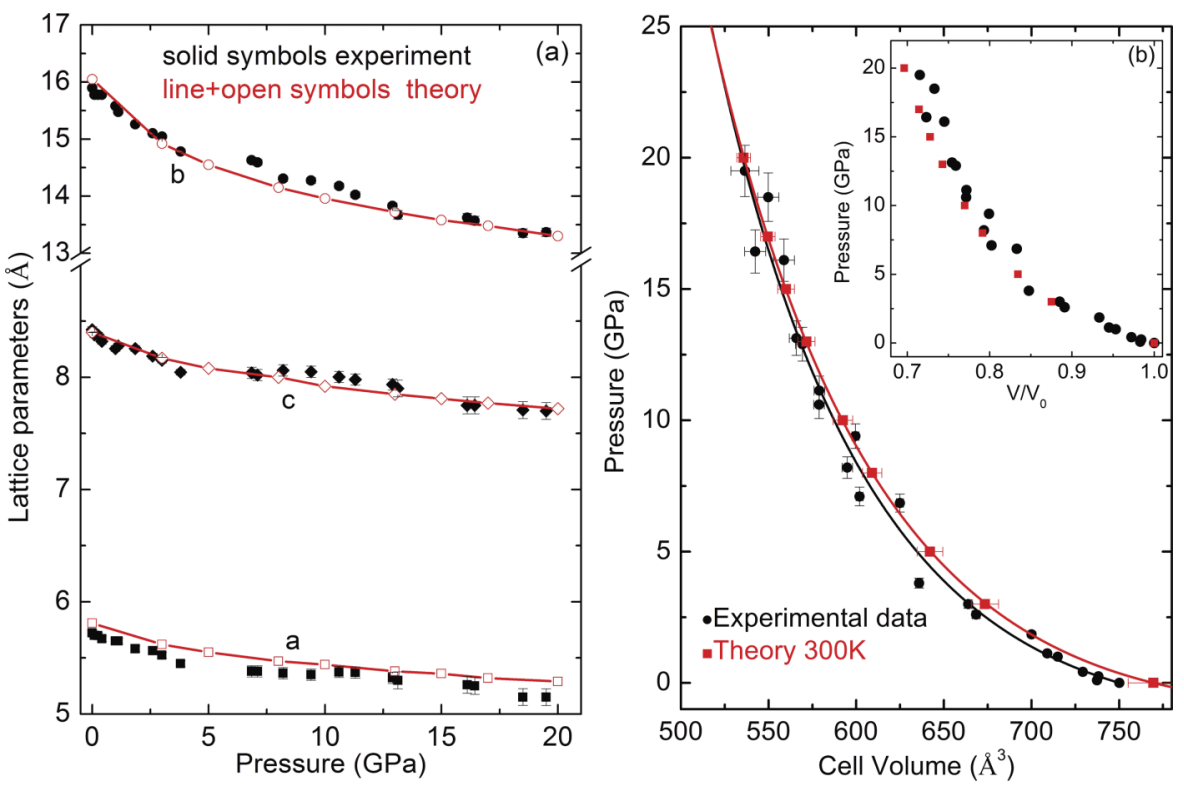

FIG. 5. Pressure dependence of (a) lattice parameters and (b) cell volume V of LLM-105. Experimental results and theoretical predictions are indicated with black and red symbols, respectively. The solid curves in (b) are the B-M 3rd order EOS fits. The inset in (b) shows the EOS data in relative volume $\left(\mathrm{V} / \mathrm{V}_{0}\right)$ representation.

highest achieved pressure (see Fig. 6(a)). Thus, there is no strong indication of a pressure or strain induced modification to the initial structure. In Figure 6(b) we can also note that our theoretically derived $\mathrm{K}_{0}$ and $\mathrm{K}^{\prime}$ values are not entirely consistent with our experimental results within $99.7 \%$ confidence levels. We believe this inconsistency is primarily due to the theoretical Vo value, which is much higher than the experimental result.

It is interesting to compare the bulk modulus $\mathrm{K}_{0}$ and its pressure derivative $\mathrm{K}_{0}^{\prime}$ of LLM-105 determined in this study with other molecular crystals. Such a comparison can provide valuable information about intermolecular interactions. ${ }^{43}$ First, $\mathrm{K}_{0}^{\prime}$ appears to be in the same range with large elemental molecules, e.g., $\mathrm{S}_{8} \mathrm{~K}_{0}^{\prime}=8.8^{44}$ and cyclic organic molecules. ${ }^{45}$ TATB, which is an insensitive high explosive cyclic molecule, exhibits $\mathrm{K}_{0}$ and $\mathrm{K}_{0}^{\prime}$ values that place well within the $50 \%$ confidence ellipse of LLM-105. ${ }^{46}$ High pressure derivative values, e.g., $\mathrm{K}^{\prime} \gg 4$ are usually representative of molecular crystals consisting of large molecules. $\mathrm{K}_{0}^{\prime}$ can be expressed as ${ }^{47}$ $\mathrm{K}_{0}^{\prime}=\mathrm{m}+\mathrm{n}+2$ in the approximation of a simple power poten- tial $\mathrm{U}(\mathrm{V})=\mathrm{A} / \mathrm{V}^{\mathrm{m}}-\mathrm{B} / \mathrm{V}^{n}$. Larger molecular size is expected to result in a higher order repulsive term and consequently to a higher $\mathrm{K}_{0}^{\prime}{ }^{43}$ On the other hand, the bulk modulus of LLM-105 appears to be much larger (almost double in experiment and in theory) in comparison to aforementioned compounds. It is plausible to attribute the higher value of $\mathrm{K}_{0}$ in the case of LLM-105 and TATB to the presence of extensive hydrogen bonding network, which may also enhance insensitivity to shock and thermal insults.

LLM-105 exhibits higher compressibility, during initial compression, along the $b$-axis as predicted by theoretical studies $^{13,14}$ and reported in a previous experimental ${ }^{8}$ study. This anisotropic compressibility is attributed to the 2D Herringbone-like pattern sheet arrangement of LLM-105 molecules perpendicular to the b-axis (Fig. 1), which results in increased repulsion between neighboring atoms in the a and $\mathrm{c}$ directions. ${ }^{13}$ This is better highlighted in the plots of the axial ratios of Fig. 7. As can be clearly seen, both $b / a$ and $b / c$ axial ratios decrease linearly up to $\approx 13 \mathrm{GPa}$. Above this pressure both axial ratios become pressure invariant up
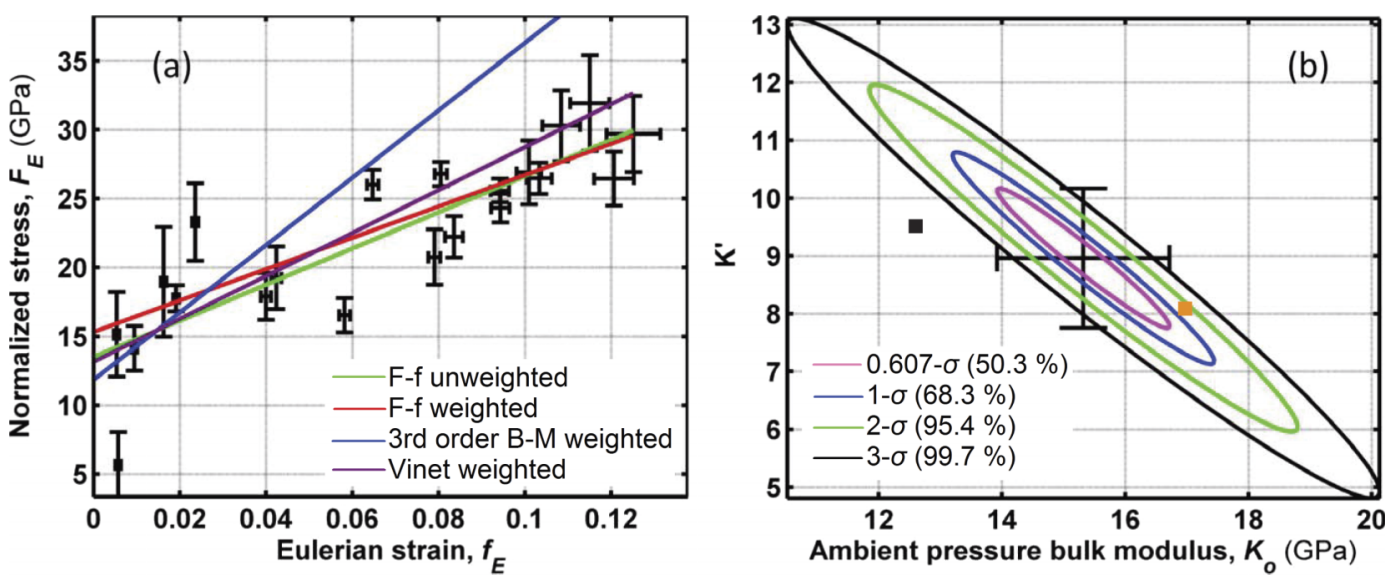

FIG. 6. (a) LLM-105 cold-compression data fits (unweighted and weighted) to a first-order F-f model and (b) plotted confidence ellipses for the weighted F-f fit. The solid black square is the result of the theoretical calculations of this study $\left(K_{0}=12.7 \mathrm{GPa}, K^{\prime}=9.4\right)$ and the solid orange square is an experimental result $\left(K_{0}=17.1 \mathrm{GPa}, K^{\prime}=8.1\right)$ for TATB. ${ }^{46}$ 
TABLE II. The most optimal EOS model experimentally weighted fits and theoretical results fit for LLM-105. Note: $K^{\prime \prime}$ (bracketed terms) is implied (see Anderson, 1995 Oxford Univ. Press ${ }^{39}$ ).

\begin{tabular}{|c|c|c|c|c|c|c|c|c|c|c|c|}
\hline \multicolumn{12}{|c|}{ Experimentally weighted fits } \\
\hline F-f order & $\mathrm{V}_{0}$ & $\mathrm{~V}_{0}$ esd & $\mathrm{K}_{0}$ & $\mathrm{~K}_{0}$ esd & $\mathrm{K}^{\prime}$ & $\mathrm{K}^{\prime}$ esd & $\mathrm{K}^{\prime \prime}$ & $\mathrm{K}^{\prime \prime}$ esd & $\chi_{\text {red }}^{2}$ & $\operatorname{Max} \Delta \mathrm{P}$ & KS-test \\
\hline 1 & 750.1000 & 1.0000 & 15.3196 & 1.3986 & 8.9599 & 1.2075 & {$[-2.1834]$} & [0.8835] & 3.9560 & 5.2977 & 0.2576 \\
\hline \multicolumn{12}{|c|}{ Theoretical results fit } \\
\hline F-f order & $\mathrm{V}_{0}$ & $\mathrm{~V}_{0}$ esd & $\mathrm{K}_{0}$ & $\mathrm{~K}_{0}$ esd & $\mathrm{K}^{\prime}$ & $\mathrm{K}^{\prime}$ esd & $\mathrm{K}^{\prime \prime}$ & $\mathrm{K}^{\prime \prime}$ esd & $\chi_{\text {red }}^{2}$ & $\operatorname{Max} \Delta \mathrm{P}$ & KS-test \\
\hline 1 & 769.5100 & 14.3600 & 12.5620 & 0.1459 & 9.5479 & 0.1340 & {$[-3.2014]$} & {$[0.1343]$} & 0.0013 & 0.0972 & 0.4860 \\
\hline
\end{tabular}

to $20 \mathrm{GPa}$. This signals a decrease in compressibility along the b-axis, which becomes equal with the other two axes. Accurate determination of the intermolecular distances as a function of pressure requires the predetermination of the atomic positional parameters at each pressure. Given the low $\mathrm{Z}$ nature of the LLM-105 molecule elements and the very high number (76) of free positional parameters this was not possible in the present study. However, the absence of both a first order phase transition and a prominent change of the Bragg peaks relative intensities with increasing pressure allows one to confidently assume that the relative arrangement and orientation of the LLM-105 molecules remain constant. With this plausible assumption, i.e., that the positional parameters remain practically constant under pressure, we have determined the intermolecular distances as a function of pressure. The Bragg peaks at (ambient conditions values) $2 \theta=3.87^{\circ} / \mathrm{d}=7.345 \AA(011)$ and $7.11^{\circ} / \mathrm{d}=4.005 \AA(012)$

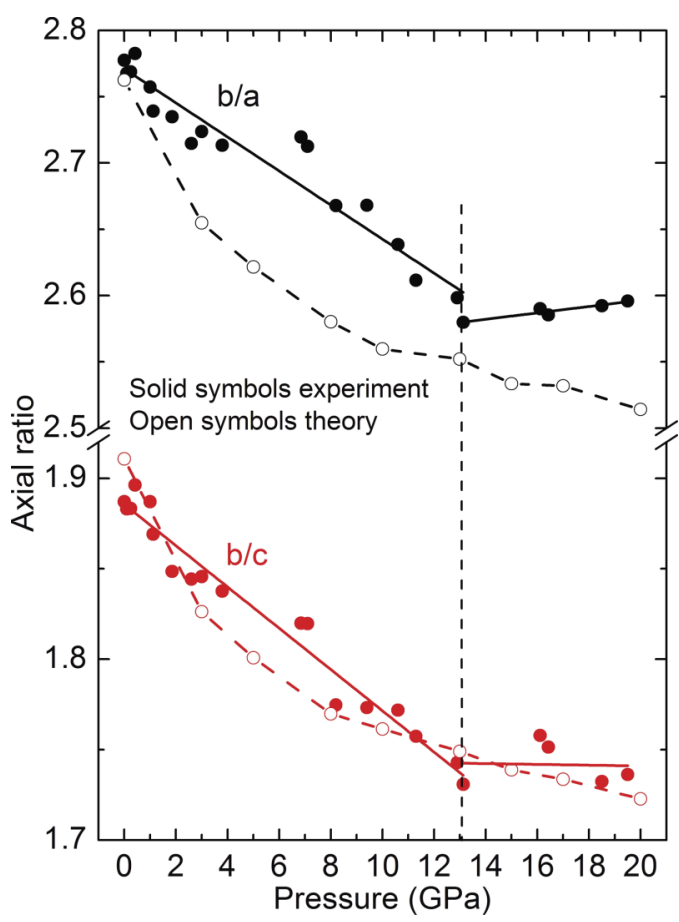

FIG. 7. Pressure dependent axial ratios $b / a$ and $b / c$ of LLM-105. Experimental results and theoretical predictions are indicated with black and red symbols, respectively. The black solid lines are linear fits of the experimental data below and above $13 \mathrm{GPa}$, and the dashed red curves are guides to the eye. The vertical dashed line highlights the pressure where the pressure dependence of the axial ratios become practically pressure invariant within scatter of experimental points. can be assigned to the ring-center intrasheet distance between in-plane adjacent LLM-105 molecules and to the periodic distance between quasi-orthogonally positioned sheets. ${ }^{6}$ It should be noted that the ring-center intrasheet distance is not the closest distance between adjacent LLM-105 molecules, but it can serve as an effective probe given the pressure independent relative orientation of the molecules. The normalized distances, determined through the pressure dependent $\mathrm{d}$ spacings of these peaks, as a function of pressure are plotted in Figure 8. Inspection of intermolecular distances reveals that the reduction of the intermolecular distances along a sheet, $9 \%$ change, is higher than that of inter-sheet out-ofplane nearly orthogonal positioned distances, $6 \%$ change. This phenomenon may be explained by higher repulsive forces acting between inter-sheet neighboring atoms resulting in a measurably lower compressibility. Here the theoretical results are not as definitive with regard to this trend given that both axial ratios continue to decrease above $13 \mathrm{GPa}$, albeit with reduced slope. One would normally expect that for a nonhydrostatic compression situation the critical pressure might be pushed to a lower pressure value because shear forces would be higher. So if the opposite effect happens then that is very interesting. Here it is the case that theory only compressed a single crystal and so shear effects are not so prevalent. Further, the calculations are conducted for a single crystal arrangement of LLM-105, whereas the experimental determinations are derived from polycrystalline powder samples.

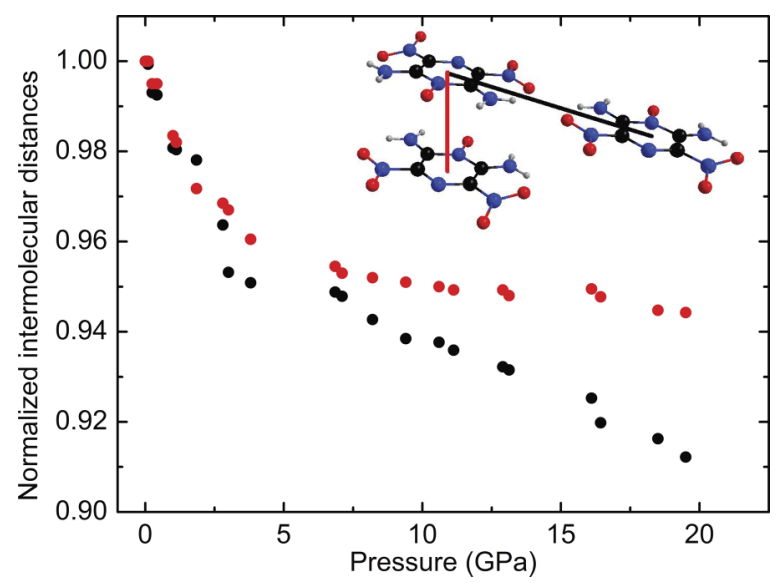

FIG. 8. Pressure dependence of the normalized LLM-1105 intermolecular intrasheet (black symbols) and intersheets distances (red symbols). See inset schematic representation for details. 


\section{CONCLUSION}

The high-pressure structural behavior of LLM-105 has been explored in a combined experimental and first-principles study up to $20 \mathrm{GPa}$. As concluded from both the X-ray diffraction measurements and the theoretical calculations, no structural phase transition has been observed up to this pressure. However, a prominent change of the compressibility of the $b$-axis relative to the other axes is observed at approximately $13 \mathrm{GPa}$, which is attributed to the decrease of the distance between molecules in adjacent in-plane sheets. The respective bulk moduli and corresponding pressure derivatives were derived from weighted and unweighted fits using selected (relatively optimal) EOS models. The EOS results will improve the confidence of thermochemical modeling predictions of high pressure-temperature detonation reactions of energetic materials such as LLM-105.

\section{ACKNOWLEDGMENTS}

This work was performed under the auspices of the U.S. Department of Energy by Lawrence Livermore National Security, LLC under Contract No. DE-AC52-07NA27344. Support from the Computational Grand Challenge Program was instrumental in conducting the computationally intensive atomistic simulations and is greatly appreciated. The experimental study was supported by the high explosives science campaign II program at Lawrence Livermore National Laboratory. The Advanced Light Source is supported by the Director, Office of Science, Office of Basic Energy Sciences, of the U.S. Department of Energy under Contract No. DE-AC0205CH11231. We thank Alastair MacDowell at Lawrence Berkeley National Laboratory for his efforts to facilitate the experiment and Jared C. Gump and Larry E. Fried for helpful discussions and support. B.K. acknowledges financial support from LLNL on Subcontract No. B608474 and additional support provided by The Scientific and Technological Research Council of Turkey fellowship under Contract No. $114 \mathrm{C} 120$.

\section{APPENDIX: COMPLETE EOS ANALYSIS AND EVALUATION PROCEDURE OF THE DIFFERENT MODELS}

In order to determine the EOS models that best represent the experimental P-V data we conducted weighted fits using Birch-Murnaghan, ${ }^{33}$ (B-M), 2 nd to 5 th orders, the Vinet, ${ }^{34}$ and the $\mathrm{F}-\mathrm{f}^{35}$ finite strain 1 st to $3 \mathrm{rd}$ order EOS models. The fit results are summarized in Table III. The "winning model" was determined using simple statistical criteria: (i) The uncertainties (errors) should be much lower than the corresponding fitting parameters. After applying this criterium 4th and 5th order BMs and 2nd and 3rd order F-fs where excluded. (ii) In the remaining EOS models, the reduced $\chi_{\text {red }}^{2}$ value closest to 1 criterium was applied. By doing so, the 3rd $\mathrm{B}-\mathrm{M}$, instead of the 2nd B-M, was determined as the most optimal B-M EOS model. Comparison between the 3rd order B-M, Vinet and 1st order F-f EOS models reveals that the F-f model gives the lowest $\chi_{\text {red }}^{2}$ values, the lowest maximum pressure deviation from the data and the KS-test values closest to 0 and so, represents the "winning model."

The $\chi_{\text {red }}^{2}$ function is used with the assumption that measured values have uncorrelated Gaussian distributed error. For the case of a small number $(\mathrm{N}<100)$ of data points, (like most high-pressure EOS studies), the uncertainty of $\chi_{\text {red }}^{2}$ values can be unacceptably large; moreover, for nonlinear fitting forms such as higher order EOS models, the "hat" matrix does not exist. In other words, there is no reliable means to compute the number of degrees of freedom (NDFs) for parameters in a nonlinear model; and further, NDFs can vary during an optimization search for a global minimum solution. For these reasons, we also conducted Kolmogorov-Smirnov tests ${ }^{37,38}$ (KS-test), i.e., compared converged model fit residuals to a Gaussian distribution with a mean value $\mu=0$ and a variance of $\sigma^{2}=1$. The bias (highest region of sensitivity) of a KStest is selected by the comparative Gaussian mean value distribution value. In some reports, the KS-test has been proven to be more robust than the reduced $\chi_{\text {red }}^{2}$ formalism. ${ }^{48} \mathrm{KS}$-test values range from 0 (optimal) to 1 (poor).

TABLE III. Parameters of EOS models derived from fits to our data weighted according to experimental uncertainties LLM-105. Note: $K^{\prime \prime}$ is implied for 1st, 2nd and 3rd B-M and F(f) $1^{\text {st }}$ order results (see: Anderson, 1995 Oxford Univ. Press).

\begin{tabular}{|c|c|c|c|c|c|c|c|c|c|c|c|c|c|}
\hline B-M order & $\mathrm{V}_{0}$ & $\mathrm{~V}_{0}$ esd & $\mathrm{K}_{0}$ & $\mathrm{~K}_{0}$ esd & $\mathrm{K}^{\prime}$ & $\mathrm{K}^{\prime}$ esd & $\mathrm{K}^{\prime \prime}$ & $\mathrm{K}^{\prime \prime}$ esd & $\mathrm{K}^{\prime \prime \prime}$ & $\mathrm{K}^{\prime \prime \prime}$ esd & $\chi_{\text {red }}^{2}$ & $\operatorname{Max} \Delta \mathrm{P}$ & KS-test \\
\hline 3 & 750.1005 & 0.2852 & 11.8363 & 1.1623 & 17.7905 & 4.5725 & {$\left[\begin{array}{lll}-17.561 & 1\end{array}\right]$} & [11.175 1] & 0 & 0 & 14.4669 & 9.2728 & 0.6214 \\
\hline 4 & 750.1694 & 0.2161 & 9.1658 & 1.5143 & 39.03517 & 13.6751 & -163.7116 & 144.4954 & 0 & 0 & 10.5734 & 7.0207 & 0.2786 \\
\hline 5 & 750.1871 & 0.1997 & 7.7683 & 2.0380 & 62.7468 & 33.6552 & -562.2674 & 746.3526 & [14050.2663] & [29812.8463] & 4.6031 & 4.8704 & 0.3433 \\
\hline & 750.0378 & 0.3275 & 13.1417 & 0.9188 & 11.9140 & 1.9647 & {$[-3.1134]$} & [0.9896] & & & 11.8954 & 10.3653 & 0.5648 \\
\hline F-f order & $\mathrm{V}_{0}$ & $\mathrm{~V}_{0}$ esd & $\mathrm{K}_{0}$ & $\mathrm{~K}_{0}$ esd & $\mathrm{K}^{\prime}$ & $\mathrm{K}^{\prime}$ esd & $\mathrm{K}^{\prime \prime}$ & $\mathrm{K}^{\prime \prime}$ esd & $\mathrm{K}^{\prime \prime \prime}$ & $\mathrm{K}^{\prime \prime \prime}$ esd & $\chi_{\text {red }}^{2}$ & $\operatorname{Max} \Delta \mathrm{P}$ & KS-test \\
\hline 1 & 750.1000 & 1.0000 & 15.3196 & 1.3986 & 8.9599 & 1.2075 & -2.18347 & 0.88357 & $\mathrm{NaN}$ & $\mathrm{NaN}$ & 3.9560 & 5.2977 & 0.2576 \\
\hline 2 & 750.1000 & 1.0000 & 13.0955 & 2.1907 & 15.3362 & 6.0774 & -14.5769 & 16.4550 & $\mathrm{NaN}$ & $\mathrm{NaN}$ & 4.1133 & 5.4831 & 0.2170 \\
\hline
\end{tabular}


${ }^{1}$ E. Stavrou, M. Ahart, M. F. Mahmood, and A. F. Goncharov, Sci. Rep. 3, 1209 (2013).

${ }^{2}$ C. M. Tarver, P. A. Urtiew, and T. D. Tran, J. Energ. Mater. 23, 183 (2005).

${ }^{3}$ T. D. Tran, P. F. Pagoria, D. M. Hoffman, B. Cunningham, J. L. Cutting, R. S. Lee, and R. L. Simpson, in Proccedings of the 12th International Detonation Symposium, San Diego, CA, August 11-16 (2002) (Office of Naval Research, 2002).

${ }^{4}$ P. F. Pagoria, A. R. Mitchell, R. D. Schmidt, R. Simpson, F. Garcia, J. W. Forbes, R. Swansiger, and D. Hoffman, "Synthesis, scale-up and characterization of 2,6-diamino-3,5-dinitropyrazine-1-oxide," Technical Report No. UCRL-JC- 130518, Lawrence Livermore National Laboratory, 1998.

${ }^{5}$ R. D. Gilardi and R. J. Butcher, Acta Crystallogr., Sect. E: Crystallogr. Commun. 57, 0657 (2001).

${ }^{6}$ J. Chen, Z. Qiao, L. Wang, F. Nie, G. Yang, and H. Huang, Mater. Lett. 65, 1018 (2011).

${ }^{7}$ J. C. Gump, C. A. Stoltz, B. G. Freedman, and S. M. Peiris, "Shock compression of condensed matter - 2009, Pts 1 and 2," AIP Conf. Proc. 1195, 541 (2009).

${ }^{8}$ J. C. Gump, C. A. Stoltz, B. P. Mason, B. G. Freedman, J. R. Ball, and S. M. Peiris, J. Appl. Phys. 110, 073523 (2011).

${ }^{9}$ M. Hai-Xia, S. Ji-Rong, Z. Feng-Qi, G. Hong-Xu, and H. Rong-Zu, Chin. J. Chem. 26, 1997 (2008).

${ }^{10}$ X. Zhao and Z. Liu, J. Chem. Res. 37, 425 (2013).

${ }^{11}$ F. Gouranlou and I. Kohsari, Asian J. Chem. 19, 4309 (2007).

${ }^{12}$ W. D. He, G. Zhou, N. B. Wong, A. M. Tian, and X. P. Long, J. Mol. Struct.: THEOCHEM 723, 217 (2005).

${ }^{13}$ M. R. Manaa, I.-F. W. Kuo, and L. E. Fried, J. Chem. Phys. 141, 064702 (2014).

${ }^{14}$ Q. Wu, C. Yang, Y. Pan, F. Xiang, Z. Liu, W. Zhu, and H. Xiao, J. Mol. Model. 19, 5159 (2013).

${ }^{15}$ W. Xu, C. An, J. Wang, J. Dong, and X. Geng, Propellants, Explos., Pyrotech. 38, 136 (2013).

${ }^{16}$ B. B. Averkiev, M. Y. Antipin, I. L. Yudin, and A. B. Sheremetev, J. Mol. Struct. 606, PII S0022 (2002).

${ }^{17}$ Y. Shen, R. S. Kumar, M. Pravica, and M. F. Nicol, Rev. Sci. Instrum. 75, 4450 (2004).

${ }^{18}$ S. Klotz, J.-C. Chervin, P. Munsch, and G. L. Marchand, J. Phys. D: Appl. Phys. 42, 075413 (2009).

${ }^{19}$ M. Matsui, J. Phys.: Conference Series 215, 012197 (2010).

${ }^{20}$ K. Syassen, High Pressure Res. 28, 75 (2008).

${ }^{21}$ A. P. Hammersley, S. O. Svensson, M. Hanfland, A. N. Fitch, and D. Hausermann, High Pressure Res. 14, 235 (1996), 4th Workshop of the IUCr
High Pressure Group on Synchrotron and Neutron Sources, KEK, JAPAN, MAR 22-24, 1995.

${ }^{22}$ J. P. Perdew, K. Burke, and M. Ernzerhof, Phys. Rev. Lett. 78, 1396 (1997).

${ }^{23}$ S. Grimme, J. Comput. Chem. 27, 1787 (2006).

${ }^{24}$ S. Goedecker, M. Teter, and J. Hutter, Phys. Rev. B 54, 1703 (1996).

${ }^{25}$ J. Schmidt, J. VandeVondele, I.-F. W. Kuo, D. Sebastiani, J. I. Siepmann, J. Hutter, and C. J. Mundy, J. Phys. Chem. B 113, 11959 (2009).

${ }^{26}$ J. VandeVondele and J. Hutter, J. Chem. Phys. 118, 4365 (2003).

${ }^{27}$ G. J. Martyna, M. E. Tuckerman, D. J. Tobias, and M. L. Klein, Mol. Phys. 87, 1117 (1996).

${ }^{28}$ S. Nose, J. Chem. Phys. 81, 511 (1984).

${ }^{29}$ G. Lippert, J. Hutter, and M. Parrinello, Mol. Phys. 92, 477 (1997).

${ }^{30} \mathrm{~J}$. VandeVondele, M. Krack, F. Mohamed, M. Parrinello, T. Chassaing, and J. Hutter, Comput. Phys. Commun. 167, 103 (2005).

${ }^{31}$ J. Gump, private communication (2014).

${ }^{32}$ A. C. Larson and R. B. Von Dreele, "General structure analysis system (GSAS)," Los Alamos National Laboratory Report No. LAUR 86-748, 2004, available at: http://www.ccp14.ac.uk/solution/gsas/ (Accessed: 10th September 2015).

${ }^{33}$ F. Birch, J. Geophys. Res. 83, 1257, doi:10.1029/JB083iB03p01257 (1978).

${ }^{34}$ P. Vinet, J. Ferrante, J. R. Smith, and J. H. Rose, J. Phys. C 19, L467 (1986).

${ }^{35}$ F. Birch, Phys. Rev. 71, 809 (1947).

${ }^{36}$ R. J. Angel, Rev. Mineral. Geochem. 41, 35 (2000).

${ }^{37}$ A. Kolmogov, Giornale dell Istitutano degli Attuari 4, 83 (1933).

${ }^{38}$ N. Smironov, Ann. Math. Stat. 19, 279 (1944).

${ }^{39} \mathrm{O}$. Anderson, Equations of State of Solids in Geophysics and Ceramic Science (Oxford University Press Inc., 1995).

${ }^{40}$ D. L. Heinz and R. Jeanloz, J. Appl. Phys. 55, 885 (1984).

${ }^{41}$ A. Polian, M. Gauthier, S. Souza, D. Trichês, J. a. Cardoso de Lima, and T. Grandi, Phys. Rev. B 83, 113106 (2011).

${ }^{42}$ B. Godwal, S. Speziale, S. Clark, J. Yan, and R. Jeanloz, J. Phys. Chem. Solids 71, 1059 (2010).

${ }^{43}$ M. A. Tuktabiev, S. V. Popova, V. V. Brazhkin, A. G. Lyapin, and Y. Katayama, J. Phys.: Condens. Matter 21, 385401 (2009).

${ }^{44}$ S. Vaidya and G. Kennedy, J. Phys. Chem. Solids 33, 1377 (1972).

${ }^{45}$ S. Vaidya and G. Kennedy, J. Chem. Phys. 55, 987 (1971).

${ }^{46}$ L. L. Stevens, N. Velisavljevic, D. E. Hooks, and D. M. Dattelbaum, Propellants, Explos., Pyrotech. 33, 286 (2008).

${ }^{47}$ I. Aleksandrov, A. Goncharov, A. Zisman, and S. Stitshov, Sov. Phys. - JETP 66, 384 (1987).

${ }^{48} \mathrm{G}$. Andrae, T. Schulze-Hartung, and P. Melchior, "Dos and don'ts of reduced chi-squared," e-print arXiv:1012.3754v1 (2010). 